\title{
Design of inductively degenerated common source RF CMOS Low Noise Amplifier
}

\author{
D MALATHI* and M GOMATHI \\ Department of Electronics and Communication Engineering, Kongu Engineering College, Perundurai 638060, \\ India \\ e-mail: malathid2001@gmail.com; gomathi.murugesan@yahoo.com
}

MS received 5 May 2015; revised 18 July 2018; accepted 31 October 2018; published online 10 December 2018

\begin{abstract}
The Low Noise Amplifier (LNA) is the first stage in RF CMOS receivers. The Common Gate (CG) LNA and Inductively Degenerated Common Source (CS) LNA are one of the widely used topologies for realizing RF CMOS receivers. The present work emphasizes a simple and exhaustive search procedure for the synthesis and analysis of CMOS CG and Inductively Degenerated CS LNA circuits. The width (W), gate source voltage $\left(V_{g s}\right)$ and drain source voltage $\left(V_{d s}\right)$ of the transistors constitute the design space in the circuit design. The design first involves the use of a circuit simulator (HSPICE) to obtain the small signal parameters of the circuit for various $\mathrm{W}, V_{g s}$, and $V_{d s}$ of the transistors and then to generate a Look-Up Table (LUT) for all design points using the obtained values. This LUT is used to meet the target performance specifications along with appropriate analytical expressions derived from the circuit in a numerical simulator (MATLAB). This will enable one to explore the whole design space quickly and fastly for arriving at the optimal values for the device dimensions, bias voltages and bias currents of the two LNA circuits. The design methodology is demonstrated by designing CG and Inductively Degenerated CS LNA circuits using $90 \mathrm{~nm}$ CMOS technology library in which Inductively Degenerated CS LNA gets high gain and low noise figure than CG LNA.
\end{abstract}

Keywords. Design space exploration; design points; CG-LNA; CS inductive degenerated LNA.

\section{Introduction}

Radio Frequency Integrated Circuits (RFIC) normally work between $300 \mathrm{MHz}$ to $30 \mathrm{GHz}$. RFIC applications include a mobile phone, WLAN, UWB, GPS and Bluetooth devices, etc. The optimal design of CMOS RF circuits that meets all target specifications is a challenging task. The success of a receiver's design is measured in multiple dimensions: receiver sensitivity, selectivity, and proclivity to reception errors. It calls for intuition, prior design experience, and timeconsuming simulations to be carried out with circuit simulators. In the design of CMOS RF circuits, the main challenge to meet the design specifications is in the optimum selection of the sizes of the various transistors and the associated DC bias levels. Given a set of device dimensions and bias voltages, circuit simulators can evaluate the performance of the circuit with very high accuracy. But the simulation time required to exhaustively explore the entire design space (all permissible devices dimensions and bias voltages) is difficult to estimate and is normally prohibitively very high.

The first stage of a CMOS RF receiver is Low Noise Amplifier (LNA). The LNA plays an undisputed

*For correspondence importance in the receiver design. Its main function is to provide enough gain to amplify extremely low signals without adding noise, thus preserving the required signal to noise ratio of the system at extremely low power levels. The design methodology for Radio Frequency (RF) CMOS LNA using current-based Metal-Oxide-Semiconductor Field-Effect Transistor (MOSFET) model was converted from weak to strong inversion regions including moderate inversion by Baroncini et al [1]. The inductor realization constraints are not considered and hence noise expressions require some corrections. The transistors that operate in moderate inversion are larger than those in strong inversion. As the integrated inductors are much bigger, the area is increased and NF of $1.4 \mathrm{~dB}$ is obtained for $2.5 \mathrm{GHz}$ frequency.

An inductorless LNA with an active balun for radio applications between $100 \mathrm{MHz}$ and $6 \mathrm{GHz}$ was proposed by Blaakmeer et al [2]. A combination of a CG stage and an admittance-scaled CS stage with replica biasing is exploited to maximize balanced operation simultaneously canceling the noise and distortion of the CG stage. NF close to or below $3 \mathrm{~dB}$ is achieved for $0.2-5 \mathrm{GHz}$ frequency and $1.2 \mathrm{~V}$ power supply and good linearity is obtained when the CSstage is carefully optimized. 
A broadband inductorless LNA design utilizing simultaneous noise and distortion cancellation is exploited by Chen et al [3]. The minimum internal gain of $14.5 \mathrm{~dB}$ and $\mathrm{NF}$ of $2.6 \mathrm{~dB}$ is achieved for $800 \mathrm{MHz}$ to $2.1 \mathrm{GHz}$ frequency and $1.5 \mathrm{~V}$ supply voltage by drawing $11.6 \mathrm{~mA}$ current.

Manstretta et al [4] extended a broadband single-ended input differential output LNA for canceling second order distortion. The second-order distortion products in the differential output are cancelled effectively by using a linear feedback from the common mode output to the singleended input. The sensitivity to process, supply voltage, and temperature variations are reduced by replica bias. The NF of $2.25 \mathrm{~dB}$ in the Very High Frequency (VHF) band and 2 to $4 \mathrm{~dB}$ in the Ultra High Frequency (UHF) band are derived for $230 \mathrm{MHz}$ to $470 \mathrm{MHz}$ frequency with the power dissipation of $7.8 \mathrm{~mW}$.

Ansari et al [5] performed a full on-chip CMOS LNA topology for cognitive radios with very low power consumption by exploiting the combination of CG stage for wideband input matching and CS stage for canceling the noise and distortion of CG stage. Both n-Channel MetalOxide Semiconductor (NMOS) and p-Channel Metal-Oxide Semiconductor (PMOS) transistors are used in CS stage for improving the Second Order Input Intercept Poin t(IIP2). The output impedance of $50 \Omega$ is achieved by paralleling a resistor with the output node. The Noise Figure (NF) of 2.3 $\mathrm{dB}$ to $2.8 \mathrm{~dB}$, Input Reflection Coefficient (S11) less than $-10 \mathrm{~dB}$, the power gain of $12 \mathrm{~dB}$ and IIP2 of $20 \mathrm{dBm}$ are obtained for the range of $50 \mathrm{MHz}$ to $10 \mathrm{GHz}$ frequency and $1 \mathrm{~V}$ power supply with power consumption of $6 \mathrm{~mW}$.

The comprehensive noise performance analysis of CMOS LNA including channel noise and induced gate noise in MOS devices was performed by Dong Feng et al [6]. A new analytical noise factor formula based on noisy two-port network theory is exhibited which shows that distributed gate resistance and other losses in series with the gate have both direct and indirect contributions to the noise factor. Design tradeoffs between power dissipation, overdrive voltage, and noise figure are balanced appropriately and high performance is achieved.

The design of CMOS CG LNAs using a design space exploration for all inversion regions, from weak to strong is investigated by Fiorelli et al [7]. The exploration is performed in terms of current consumption, gain and noise figure in the design space $(I D, g m / I D)$ and the short channel effects are neglected, hence results measured are inaccurate. For smaller NF, transistor works in strong inversion and hence current consumption increases and for higher NF it is vice versa.

A $0.13 \mu \mathrm{m}$ CMOS Balun-LNA for ultra-wideband applications is investigated by Reddy et al [8] using a common gate amplifier with source degenerated inductance for input matching. The balanced operation is maximized and noise of the CG stage is cancelled by emphasizing the combination of a CG stage and an admittance-scaled CS stage with replica biasing. The $\mathrm{NF}<2.8 \mathrm{~dB}, \mathrm{~S} 11$ less than $-10 \mathrm{~dB}$ and maximum gain of $17.8 \mathrm{~dB}$ is obtained for 2.1 GHz-9.5 GHz frequency and $1.2 \mathrm{~V}$ supply with a power consumption of $18.5 \mathrm{~mW}$.

Tulunay et al [9] reported a stand-alone design automation tool tailored for RF CMOS LNA designs to obtain faster optimization. Design exploration is emphasized using circuit simulator which is time-consuming. NF less than $2.78 \mathrm{~dB}$ is measured for $900 \mathrm{MHZ}$ frequency and $2.5 \mathrm{~V}$ power supply. An automatic synthesis tool for Radio Frequency Integrated Circuits (RFIC) design is demonstrated by Tulunay et al [10]. The tool incorporated built-in numerical simulators for fast evaluation of the performance metrics. The duration of the overall optimization process is reduced which eliminates the need for circuit simulators. Nonlinearity is modelled using Volterra series method and additionally provides the on-chip inductors. NF of less than $0.75 \mathrm{~dB}$ is derived for $900 \mathrm{MHz}$ frequency and $3.3 \mathrm{~V}$ power supply.

The rest of the paper is organized as follows. Section 2 describes the proposed design methodology. Section 3 discusses the simulation results that validate the proposed design methodology. Finally, section 4 provides the conclusions of the present work.

\section{Proposed design methodology}

The design of CMOS LNA circuits using small signal models has been widely used but has some limitations in its accuracy. For example, the small signal model approach used by Tuluna et al [10] with reverse isolation is not valid for all LNA designs. Another small signal model approach is used by the BSIM3v3 [11] model considers reverse isolation. However, the extraction of the values of the associated model parameters is not straightforward. These limitations can be overcome by the proposed design.

\subsection{Small signal parameters extraction}

In RF CMOS circuits, the transistors are normally designed with large widths. For the fixed length of the transistor, the small signal parameters are obtained for different bias voltages for a particular width. This procedure is repeated for various widths. In the present work, the small signal parameters are extracted using circuit simulators (HSPICE). The parasitic capacitors associated with all the terminals of the transistor are modeled as frequency dependent current sources as described in figure 1. The various current sources of figure 1 can be expressed as follows.

$$
\begin{gathered}
I_{d s}=g_{m} V_{g s}+g_{m b s} V_{b s}+g_{d s} V_{d s} \\
I_{g}(s)=s\left(C_{g g} V_{g}+C_{g s} V_{s}+C_{g b} V_{b}+C_{g d} V_{d}\right)
\end{gathered}
$$




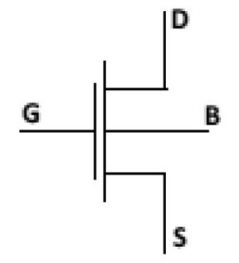

(a)

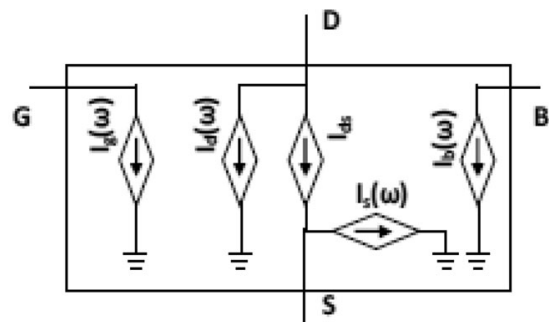

(b)
Figure 1. CMOS transistor (a) symbol, (b) proposed small signal model.

$$
\begin{gathered}
I_{d}(s)=s\left(C_{d g} V_{g}+C_{d s} V_{s}+C_{d b} V_{b}+C_{d d} V_{d}+C_{j d} V_{d b}\right) \\
I_{s}(s)=s\left(C_{s g} V_{g}+C_{s s} V_{s}+C_{s b} V_{b}+C_{s d} V_{d}+C_{j s} V_{s b}\right) \\
I_{b}(s)=s\left(C_{b g} V_{g}+C_{b s} V_{s}+C_{b b} V_{b}+C_{b d} V_{d}\right)
\end{gathered}
$$

In Eqs. (1)-(5), $g_{m}, g_{m b s}, g_{d s}$ are the gate to source transconductance, bulk to source transconductance and drain to source transconductance, respectively. $V_{g s}, V_{b s}$, $V_{d s}, V_{s b}$ represent gate to source voltage, bulk to source voltage, drain to source voltage and source to bulk voltage, respectively. Similarly, $V_{g}, V_{b}, V_{s}, V_{d}$ are gate voltage, bulk voltage, source voltage and drain voltage with respect to ground, respectively. The symbol $C_{x y},(\mathrm{x}, \mathrm{y}=\mathrm{g}, \mathrm{d}, \mathrm{s}, \mathrm{b})$ represents the capacitance relating the rate of change of charge in x terminal respect to change in voltage in y terminal. $C_{j d}$, $C_{j s}$ represent the junction capacitance at the drain and source terminals, respectively.

The transistor's small signal parameters depend directly on width, length and bias voltage at the source, drain, bulk and drain terminals. In this work, the transistors are designed at the fixed length, fixed source and bulk voltages and hence small signal parameters depend only on the width and varying gate and drain bias voltages. Thus the three parameters, namely width $(\mathrm{W})$, the gate-source voltage $\left(V_{g s}\right)$ and drain source voltage $\left(V_{d s}\right)$ of the transistors constituting the design points in the circuit that forms the design space. A LUT is created over different bias voltages for different width with the fixed length of the transistor. The LUT contains the various device transconductances, parasitic capacitances and other DC quantities like drain source current. During the design exploration procedure, this LUT is used by the MATLAB.

\subsection{Steps to explore the design space}

For determining the performance analysis of the LNA circuits the appropriate analytical expressions are derived. The analytical expressions are then used in MATLAB along with the created LUT to explore the design space and to arrive at the feasible design point. The steps to explore the design space are as follows: 2.2a Creation of LUT: For the chosen fixed transistor length and particular width, LUT is created using the obtained DC and small signal parameters. This is carried out by performing DC simulation in HSPICE for various $V_{g s}$ and $V_{d s}$.

2.2b Defining the design space: For the circuit topology, the design points are $\mathrm{W}, V_{g s}$, and $V_{d s}$ of all transistor. The size of the design space depends on the number of transistors used.

2.2c Deriving the analytical expressions: For the circuit under the considerations, the analytical expressions for determining the performance of LNA are derived using the proposed small signal model of the circuit. The analytical expressions derived include Input Reflection Coefficient (S11), Forward Gain (S21), and Noise Figure (NF).

2.2d Exploring the design space: The performances of the circuit are analyzed from the derived analytical expression in MATLAB by loading LUT. Save the design points that satisfy the performance requirement as feasible design points.

2.2e Select the optimal design: Sort the feasible design points obtained in Step 4 above with optional additional constraints on the performance and select the best possible design.

The above design space exploration is also depicted in the form of a flowchart in figure 2 .

\subsection{Design of LNA circuits}

Two important specifications for an LNA are a power impedance match and low noise figure. The NF of an LNA using MOSFETs can be made low by increasing the transconductance $\left(g_{m}\right)$ of the device and with proper layout. The source impedance that yields minimum NF is inductive in character and generally unrelated to the conditions that maximize power transfer $[12,13]$. Furthermore, the gate (input impedance) of a MOSFET is predominantly capacitive by providing a good match to a $50 \Omega$ source without degrading noise performance. Since presenting a known resistive impedance to the external world is an almost critical requirement of LNAs.

The circuit topologies that provide resistive impedance are

- Resistive termination

- Series-shunt feedback

- Common gate connection

- Inductor degeneration

2.3a Common gate LNA design: The proposed methodology is exhausted on commonly used inductor less CG LNA as in figure 3 and its small signal model is in figure 4. Small signal parameters and LUT has been created using $90 \mathrm{~nm}$ CMOS technology in HSPICE. Next the design points W, 


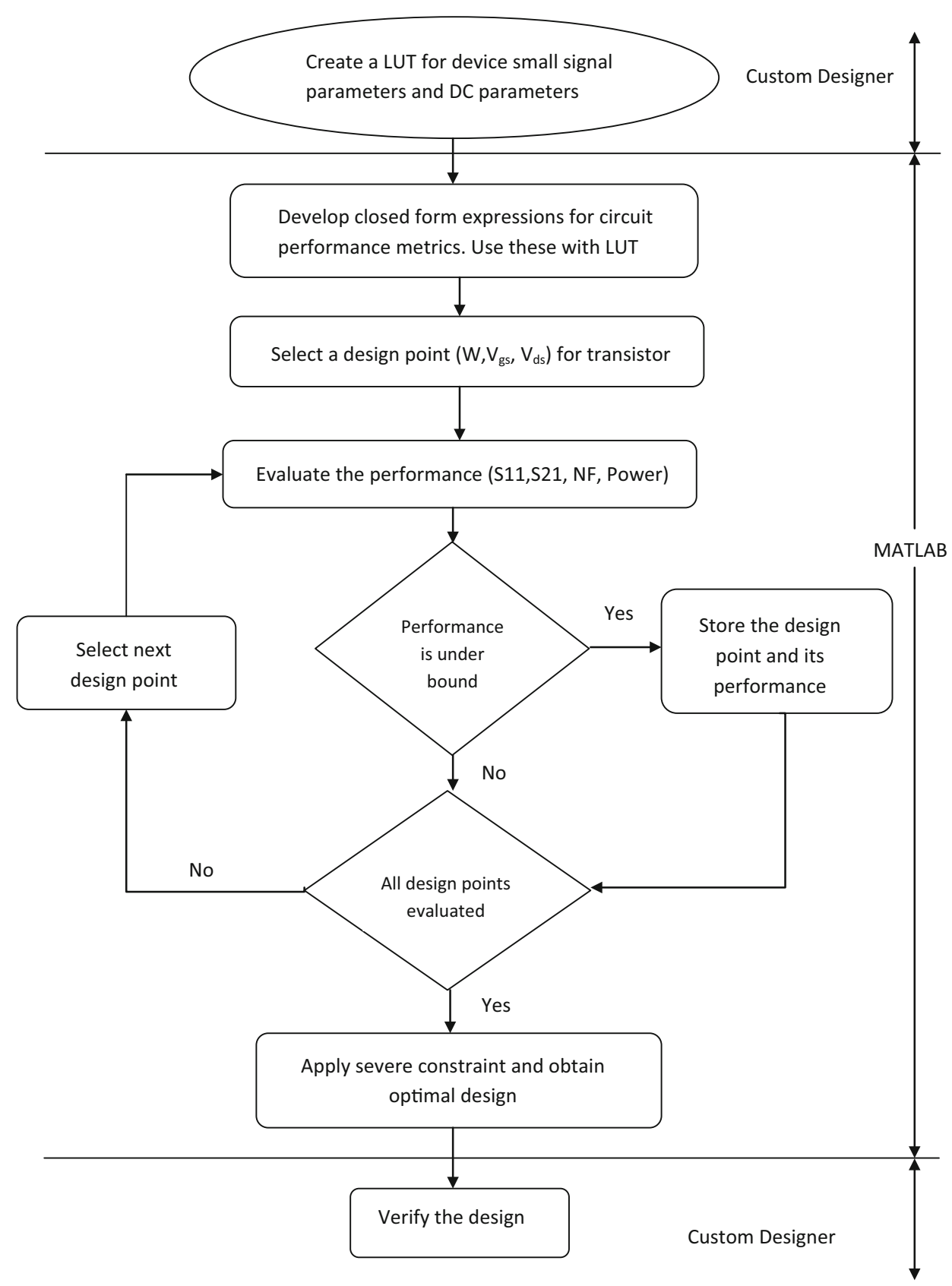

Figure 2. Flow Chart for Design Space Exploration of LNA circuits.

$V_{g s}$, and $V_{d s}$ are defined for each transistor in the circuit. The dimension of the design space is three for CG.

The input is applied to source terminal MOS and the output is taken from the drain terminal. The drain terminal is connected to the power supply through the load resistor. By properly choosing $\mathrm{W}, V_{g s}$ and $V_{d s}$ voltage and load resistor the input is amplified and is obtained at the output.

With reference to figure 4, the parasitic dependent Input Impedance of the CG LNA can be expressed as,

$$
Z_{\text {in }}(s)=\frac{a_{1}+s a_{2}}{b_{1}+s b_{2}+s^{2} b_{3}}
$$

where,

$$
\begin{gathered}
a_{1}=1+g_{d s} * R_{L} \\
a_{2}=C_{D} * R_{L}+C_{D B} * R_{L} \\
b_{1}=g_{m}+g_{m b s}+g_{d s} \\
b_{2}=G_{m} * C_{D B} * R_{L}+G_{m} * C_{D} * R_{L}+g_{d s} * C_{d g} * R_{L} \\
-C_{D B} * C_{d g} * R_{L}-C_{D} * C_{d g} * R_{L}+C_{B B} a_{1} \\
b_{3}=C_{d g} * R_{L}\left(C_{D B}+C_{D}\right)+C_{B B} * R_{L}\left(C_{D B}+C_{D}\right)
\end{gathered}
$$




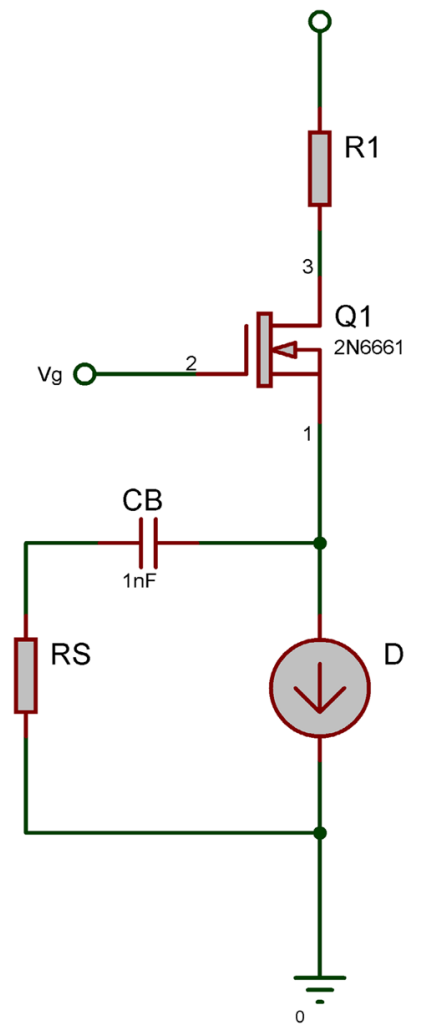

Figure 3. Common gate LNA.

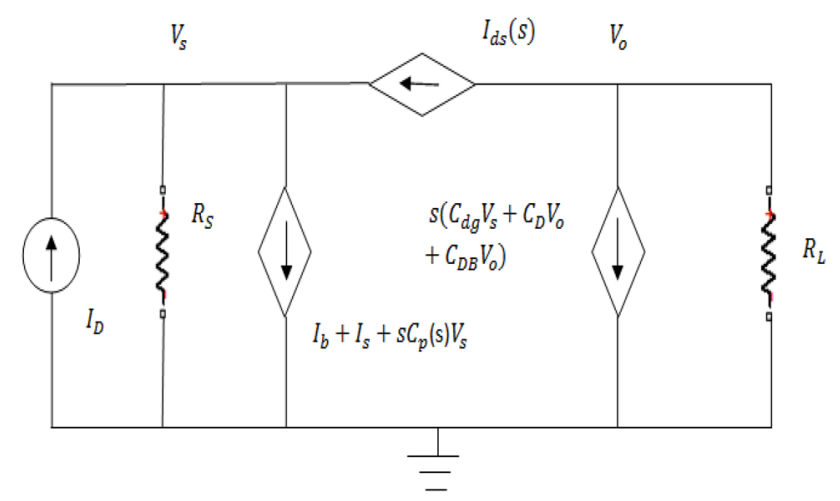

Figure 4. Small signal model of CG LNA.

$$
\begin{gathered}
G_{m}=g_{m}+g_{d s}+g_{m b s} \\
C_{D B}=C_{d b}+C_{d s}-C_{j d} \\
C_{B B}=C_{s s}+C_{s b}+C_{b s} \\
C_{D}=C_{d d}+C_{j d}
\end{gathered}
$$

Given $Z_{i n}(s)$ as in (6), the Input Reflection Coefficient can be expressed as,

$$
S_{11}(s)=\frac{Z_{\text {in }}(s)-R_{s}}{Z_{\text {in }}(s)+R_{s}}
$$

In a similar manner, the Forward Gain can be expressed as,

$$
S_{21}(s)=\frac{c_{1}-s c_{2}}{d_{1}+s d_{2}+s^{2} d_{3}}
$$

where,

$$
\begin{gathered}
c_{1}=G_{m} \\
c_{2}=C_{d g} \\
d_{1}=g_{d s}+\left(1 / R_{L}\right)-\left(G_{m} *\left(R_{S} / R_{L}\right)\right) \\
d_{2}=g_{d s} * R_{L}\left(-C_{d g}-C_{B B}+C_{p}\right)+C_{D B}+C_{D}-C_{B B} \\
*\left(R_{S} / R_{L}\right)-G_{m} * C_{D B} * R_{L}-G_{m} * C_{D} * R_{L}-G_{m} \\
*\left(R_{S} / R_{L}\right) \\
d_{3}=C_{D B} * R_{S}\left(-C_{B B}+C_{p}\right)+C_{p} * C_{D} * R_{S}-C_{B B} * C_{D}
\end{gathered}
$$

Noise Factor $(\mathrm{F})$ is also defined as,

$$
F=\frac{\text { total output noise power }}{\text { output noise due to input source }}
$$

The NF is F expressed in decibels. With reference to figure 5 , the $\mathrm{F}$ is given by,

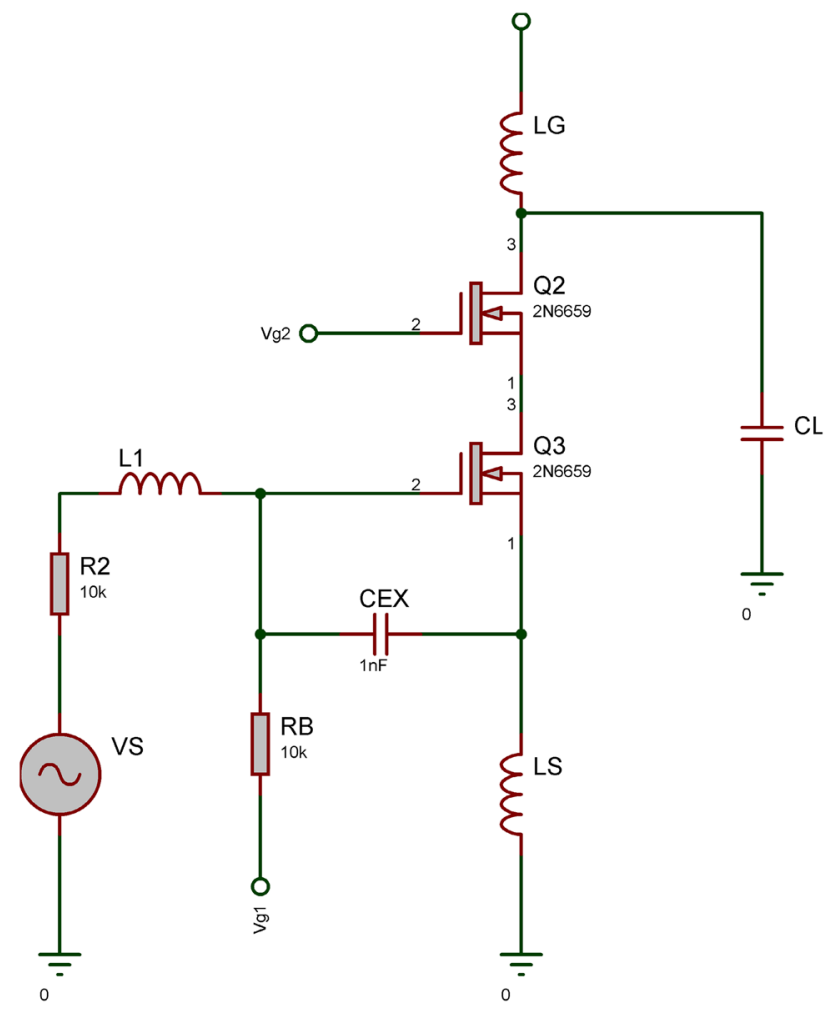

Figure 5. Inductively degenerated common source LNA. 


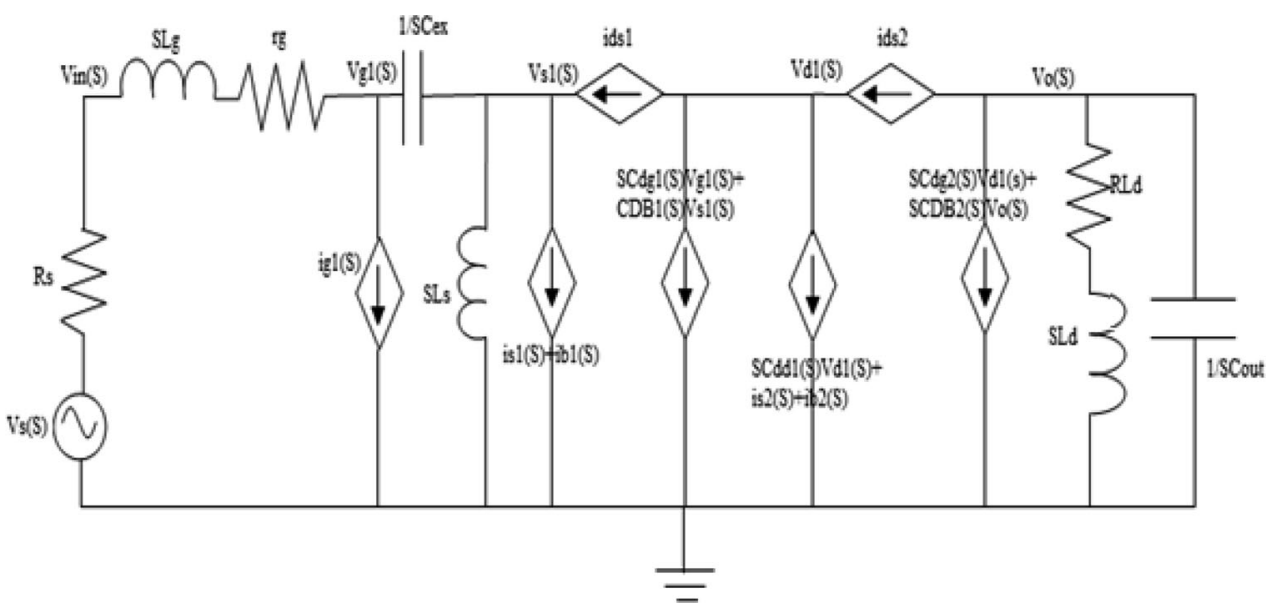

Figure 6. Small Signal Model of Inductively degenerated Common Source LNA.

$$
\begin{aligned}
F= & 1+\frac{\left(\left(1 / R_{S}\right)+s\left(C_{d g}+C_{B B}+C_{p}\right)\right)^{2} * \overline{i_{d}^{2}}}{\left(G_{m}-s C_{d g}\right)^{2} * \overline{i_{S}^{2}}} \\
& +\frac{\left(\left(1 / R_{S}\right)+G_{m}+s\left(C_{B B}+C_{p}\right)\right)^{2} * \overline{i_{L}^{2}}}{\left(G_{m}-s C_{d g}\right)^{2} * \overline{i_{S}^{2}}}
\end{aligned}
$$

where,

$$
\begin{gathered}
\overline{i_{d}^{2}}=4 k T\left(\frac{2}{3} g_{m}\right) \Delta f \\
\overline{i_{L}^{2}}=4 k T \frac{1}{R_{L}} \Delta f \\
\overline{i_{S}^{2}}=4 k T \frac{1}{R_{S}} \Delta f
\end{gathered}
$$

The NF of the circuit is given by,

$$
N F=10 * \log (F)
$$

2.3b Inductively degenerated common source LNA: Inductively degenerated common source LNA is most commonly used in narrowband applications because it has resistorless input impedance for having low noise figure. To have proper impedance matching inductor is used in the source of the M1 transistor. Capacitor $C_{e x}$ is used to adjust the Qpoint of the transistor. The circuit for inductively degenerated common source LNA is shown in figure 5. The input is applied to source terminal M1 and the output is taken from the drain terminal of M2. The drain terminal of M2 is connected to the power supply through the load resistor. By properly choosing $\mathrm{W} 1, \mathrm{~W} 2, V_{g s 1}, V_{d s 1}, V_{g 2}$ transistor the input is amplified and is obtained at the output.

Similar to CG LNA the analytical expressions for Input Impedance, Forward Gain and Noise Figure of Inductively Degenerated CS LNA are derived using the small signal model given in figure 6 . The parasitic dependent Input
Impedance of Inductively Degenerated CS LNA can be expressed as,

$$
\operatorname{Zin}(s)=\operatorname{Zin} 1(s)+r g+\left(s * \mathrm{~L}_{\mathrm{g}}\right)
$$

In a similar manner, the Forward Gain of Inductively Degenerated CS LNA can be expressed as,

$$
S_{21}(s)=\left(\frac{1}{\mathrm{R}}\right) *\left(\frac{n e v}{d e v}\right)
$$

The F of Inductively Degenerated CS LNA is given by,

$$
F=1+\frac{V o 2}{V o 1}+\frac{V o 3}{V o 1}+\frac{V o 4}{V o 1}
$$

From these equations, the Input Reflection Coefficient, Forward Gain and Noise Figure of Inductively Degenerated CS LNA is calculated for all design points and its optimized results are in table 3 .

The design points were identified with the sweep of design variables. For the Common Gate model, the widths of the transistors $\mathrm{M}$ range from $10 \mu \mathrm{m}$ to $300 \mu \mathrm{m}$ in a step of $50 \mu \mathrm{m}$. The drain to source voltages ranges from $0.2 \mathrm{~V}$ to $1.6 \mathrm{~V}$ and gate to source voltages ranges from $0.4 \mathrm{~V}$ to $1.7 \mathrm{~V}$. These ranges chosen for the free parameters are considered large enough to cover all feasible design cases.

For the Inductively Degenerated Common Source LNA, the width of the transistor M1 ranges from $50 \mu \mathrm{m}$ to $400 \mu \mathrm{m}$ in a step of $50 \mu \mathrm{m}$. The drain to source voltages of transistor M1 ranges from $0.2 \mathrm{~V}$ to $1.6 \mathrm{~V}$, the gate to source voltages of transistor $\mathrm{M} 1$ ranges from $0.4 \mathrm{~V}$ to $1.7 \mathrm{~V}$ and the gate voltage of $\mathrm{M} 2$ transistor ranges from $0.5 \mathrm{~V}$ to $1.8 \mathrm{~V}$. The design constraints are chosen for the CG LNA and Inductively Degenerated CS LNA are given in Table 1. Under these constraints, the feasible points were obtained using LUT in conjunction with analytical expressions in MATLAB by performing an exhaustive search among various design points. 
Table 1. Design specifications.

\begin{tabular}{lc}
\hline Design specifications & Value \\
\hline Supply voltage $\left(V_{d d}\right)$ & $1.8 \mathrm{~V}$ \\
Input reflection $\left(S_{11}\right)$ & $<-10 \mathrm{~dB}$ \\
Forward gain $\left(S_{21}\right)$ & $>10 \mathrm{~dB}$ \\
Noise figure $(\mathrm{NF})$ & $<5 \mathrm{~dB}$ \\
Bias current $\left(I_{d s}\right)$ & $<25 \mathrm{~mA}$ \\
Resonant frequency & $2 \mathrm{GHz} / 10 \mathrm{GHz}$ \\
Source impedance & $50 \Omega$ \\
Load impedance & $5 \mathrm{pF}$ \\
CMOS technology & $90 \mathrm{~nm}$ \\
\hline
\end{tabular}

\section{Results and discussion}

The RF CMOS CG LNA is designed using $90 \mathrm{~nm}$ CMOS technology in HSPICE and simulated using MATLAB. The number of design points in this space exploration process is defined by the number of free parameters, the step sizes of the free parameters and their respective minimum and maximum value. In this work $\mathrm{W}, V_{g s}$ and $V_{d s}$ of the transistor were used as design points for the CG LNA design explorations and W1, W2, $V_{g s 1}, V_{d s 1}, V_{g 2}$ of the transistors were used as design points for inductively degenerated CS LNA. Each design point is validated in less than a millisecond using any circuit simulator. The time required to arrive at the feasible design points using a circuit simulator alone will be very high, and in addition, it would require intense manual effort.

The effective use of LUT obtained from HSPICE in conjunction with MATLAB completes the search and arrives at the feasible results of CG LNA and inductively degenerated CS LNA within a few seconds. The size of the search space increases as the number of design points increases which in turn increases as the number of transistors in the circuit increase, and this leads to an increase in the simulation.

The small signal parameters are obtained for various design points ( $\mathrm{W}, V_{g s}$, and $V_{d s}$ ). Look Up Table is formed for various design points using the obtained small signal parameters from the design as shown in figure 7. The highlighted values in figure 7 are considered for the design.

The small signal parameters are obtained for various design points (W1, W2, $V_{g s 1}, V_{d s 1}, V_{g 2}$ ). Look up table is formed for various design points using the obtained small signal parameters as shown in figure 8. Tables 2 and 3 contain the feasible design points of CG LNA and Inductively Degenerated CS LNA, respectively obtained by MATLAB for operating frequency of $2 \mathrm{GHz}$. Each row in tables 2 and 3 corresponds to the feasible design points optimized in any single objective. The highlighted values in figure 8 are considered for the design. The design parameters are global since the operating frequency and supply voltage is common to the entire circuit. The Gate source voltage and drain source voltage is maintained globally.

\subsection{Parasitic effects}

Inductive degeneration is popular because of package parasitics. The parasitics in LNA design can be reduced during package. This requires good model pf package and bond wires. The inductance of the input loop depends on the arrangement of bond wires, die size and pad locations. The

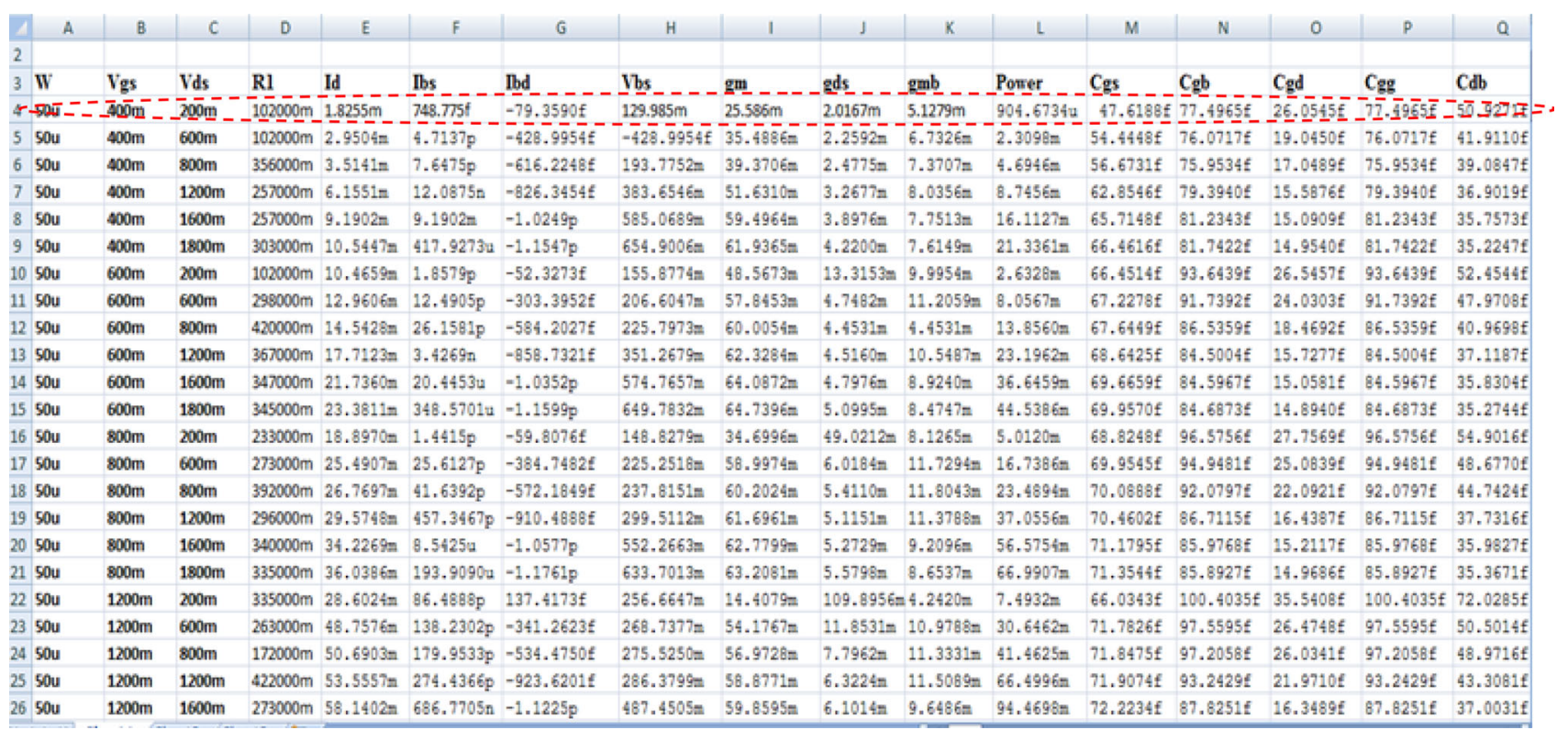

Figure 7. Look up table using small signal parameters of CG LNA. 


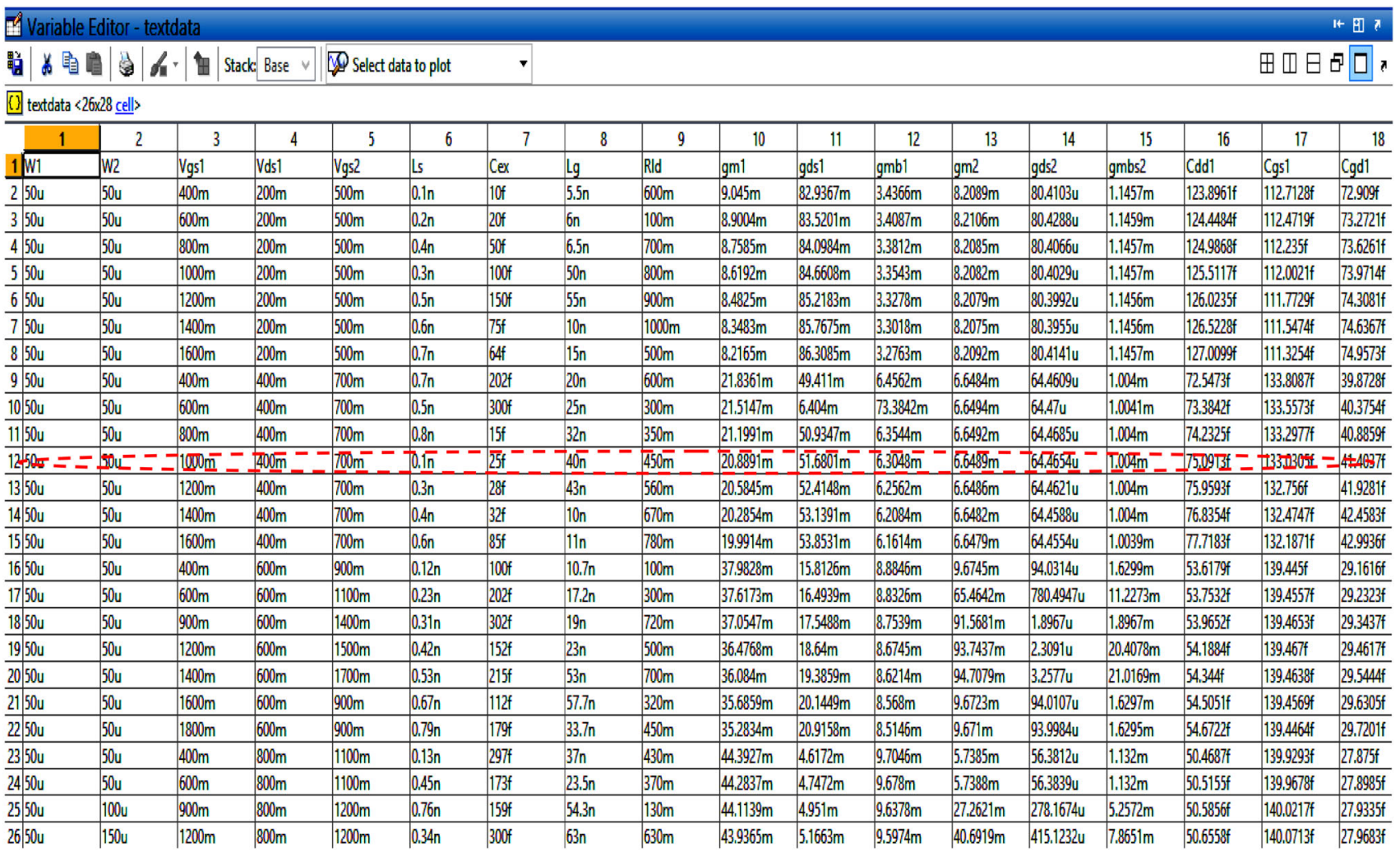

Figure 8. Look UP Table using small signal parameters of inductively degenerated CS LNA.

Table 2. Design result of CG LNA at $\mathrm{F}=2 \mathrm{GHz}$.

\begin{tabular}{|c|c|c|c|c|c|}
\hline Sl.No & $\mathrm{W}(\mu \mathrm{m})$ & $\mathrm{V}_{\mathrm{gs}}(\mathrm{mV})$ & $\mathrm{V}_{\mathrm{ds}}(\mathrm{mV})$ & $\mathrm{R}_{\mathrm{L}}(\Omega)$ & Optimized parameters and its value \\
\hline 1. & 50 & 400 & 200 & 102 & Minimum Power - $904.6734 \mu \mathrm{W}$ \\
\hline 2. & 50 & 600 & 800 & 420 & Maximum S21 - 13.6333 dB \\
\hline 3. & 300 & 1600 & 1800 & 397 & Minimum NF - 0.7798 dB \\
\hline 4. & 50 & 400 & 200 & 102 & Minimum S11 - $17.5212 \mathrm{~dB}$ \\
\hline
\end{tabular}

Table 3. Design result of inductively degenerated CS LNA at F $=2 \mathrm{GHz}$.

\begin{tabular}{|c|c|c|c|c|c|c|c|c|c|c|c|c|}
\hline S1.No & $\begin{array}{l}\text { W1 } \\
(\mu \mathrm{m})\end{array}$ & $\begin{array}{l}\mathrm{W} 2 \\
(\mu \mathrm{m})\end{array}$ & $\begin{array}{l}\mathrm{V}_{\mathrm{gs} 1} \\
(\mathrm{mV})\end{array}$ & $\begin{array}{l}\mathrm{V}_{\mathrm{ds} 1} \\
(\mathrm{mV})\end{array}$ & $\begin{array}{l}\mathrm{V}_{\mathrm{ds} 2} \\
(\mathrm{mV})\end{array}$ & $\begin{array}{c}\mathrm{L}_{\mathrm{s}} \\
(\mathrm{nH})\end{array}$ & $\begin{array}{l}\text { Cex } \\
(\mathrm{pF})\end{array}$ & $\begin{array}{c}\mathrm{L}_{\mathrm{g}} \\
(\mathrm{nH})\end{array}$ & $\begin{array}{l}\text { Rld } \\
(\Omega)\end{array}$ & $\mathrm{Ld}(\mathrm{nH})$ & $\begin{array}{l}\mathrm{Rg} \\
(\Omega)\end{array}$ & $\begin{array}{c}\text { Optimized parameters and } \\
\text { its value }\end{array}$ \\
\hline 1. & 300 & 30 & 700 & 400 & 700 & 0.54 & 101.5 & 63.1 & 0.64 & 0.01259 & 0.2782 & $\begin{array}{c}\text { Minimum Power }-1.0353 \\
\mathrm{~mW}\end{array}$ \\
\hline 2. & 250 & 150 & 900 & 1200 & 1500 & 0.13 & 89 & 32.7 & 0.51 & 0.01223 & 0.3328 & Maximum S21 - 31.9719 \\
\hline 3. & 100 & 150 & 1200 & 700 & 1200 & 0.24 & 202.5 & 60.2 & 0.37 & 0.01206 & 0.8285 & Minimum NF $-0.0630 \mathrm{~dB}$ \\
\hline 4. & 50 & 50 & 1000 & 400 & 700 & 0.1 & 25 & 40 & 0.45 & 0.01253 & 1.6645 & $\begin{array}{c}\text { Minimum S11 }-24.3172 \\
\mathrm{~dB}\end{array}$ \\
\hline
\end{tabular}

changing flux generates an EMF around the circuit loop and it causes undesired mutual inductance to other parts of the circuit. Many designs also require ESD protection which manifests as increased capacitance on the pads.
Figure 9 shows the comparison of CG LNA and Inductively Degenerated CS LNA at $\mathrm{F}=2 \mathrm{GHz}$. The performance of Inductively Degenerated CS LNA is high compared to CG LNA. 


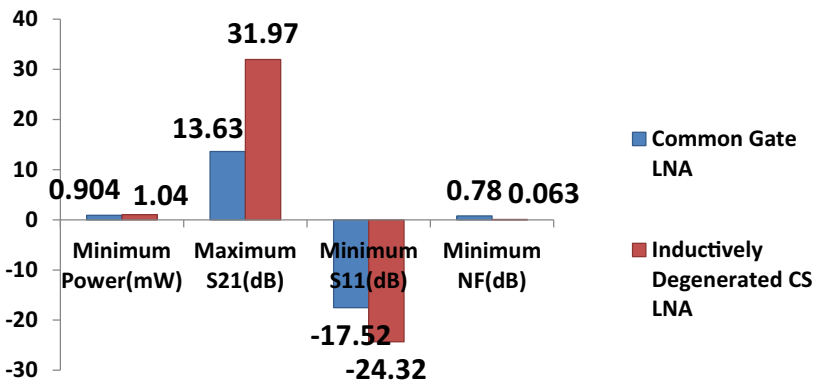

Figure 9. Comparison of CG LNA and Inductively Degenerated CS LNA at $\mathrm{F}=2 \mathrm{GHz}$.

\section{Conclusion}

The RF CMOS CG LNA and Inductively Degenerated CS LNA is designed for space exploration and the synthesis is carried out by an exhaustive search to obtain the feasible design points which provide minimum power, maximum gain, minimum noise figure, and minimum input reflection coefficient at a $2 \mathrm{GHz}$ frequency. In this, CS Inductively Degenerated LNA is high in performance compared to CG LNA with $57.35 \%$ of the increase in gain and $91 \%$ of reduced noise figure.

\section{References}

[1] Baroncini, Virgínia Helena Varotto and Oscar da Costa Gouveia-Filho 2004 Design of RF CMOS low noise amplifiers using a current based MOSFET model. In: Proceedings of International Symposium on Integrated circuits and system design 82-87

[2] Blaakmeer, Stephan C, Eric A M Klumperink, Domine M W Leenaerts and Bram Nauta 2008 Wideband Balun-LNA with simultaneous output balancing, noise-canceling and distortion-canceling. IEEE Journal of Solid-State Circuits 43: $1341-1350$

[3] Chen, Wei-Hung, Gang Liu, Boos Zdravko and Ali M Niknejad 2008 A highly linear broadband CMOS LNA employing noise and distortion cancellation. IEEE Journal of Solid-State Circuits 43(5): 1164-1176

[4] Manstretta and Danilo 2012 A broadband low-power lownoise active balun with second-order distortion cancellation. IEEE Journal of Solid-State Circuits 47: 407-420

[5] Ansari, Amirhossein and Mohammad Yavari 2011 A very wideband low noise amplifier for cognitive radios. In: Proceedings of 18th IEEE Int Conf. On Electronics, Circuits and System: 623-626

[6] Feng, Dong and Bingxue Shi 2005 Comprehensive analysis and optimization of CMOS LNA noise performance. In: Proceedings of Asia and South Pacific Design Automation Conference: 1204-1207

[7] Fiorelli, Rafaella and Fernando Silveira 2008 Common gate LNA design space exploration in all inversion regions. Micro-Nanoelectronics, Technology and Applications 119-122

[8] Reddy, Prakash B S and Bhuvan B 2012 Design of ultrawideband balun-low noise amplifiers. In: Students Conference on Electrical, Electronics and Computer Science 1-4

[9] Tulunay, Gülin and Sina Balkir 2008 A synthesis tool for CMOS RF low-noise amplifiers. IEEE Transactions on Computer-Aided Design of Integrated Circuits and Systems 27: 977-982

[10] Tulunay, Gülin and Sina Balkir 2006 Automatic synthesis of CMOS RF front-ends. In: Proceedings of IEEE International Symposium on Circuits and System: 880-883

[11] BSIM3v3 Manual

[12] Fahimnia Mohammad-Taheri M, Wang Y, Yu M and SafaviNaeini S 2010 An Efficient Method to Design Optimum Millimeter Wave Low Noise Amplifier. In: Proceedings of IEEE International Symposium on Antennas and Propagation Society: 1-4

[13] Shaeffer, Derek K and Thomas H Lee 1997 A 1.5-V, 1.5$\mathrm{GHz}$ CMOS low noise amplifier. IEEE Journal on SolidState Circuits 32: 745-759 